\title{
Angular magnetoresistance oscillations in bilayers in tilted magnetic fields
}

\author{
Victor M. Yakovenko ${ }^{1}$, Benjamin K. Cooper \\ Condensed Matter Theory Center, Department of Physics, University of Maryland, College Park, MD 20742-4111, USA
}

\begin{abstract}
Angular magnetoresistance oscillations (AMRO) were originally discovered in organic conductors and then found in many other layered metals. It should be possible to observe AMRO to semiconducting bilayers as well. Here we present an intuitive geometrical interpretation of AMRO as the Aharonov-Bohm interference effect, both in real and momentum spaces, for balanced and imbalanced bilayers. Applications to the experiments with bilayers in tilted magnetic fields in the metallic state are discussed. We speculate that AMRO may be also observed when each layer of the bilayer is in the composite-fermion state.
\end{abstract}

Key words: Magnetoresistance oscillations, Bilayers, Interlayer tunneling, Aharonov-Bohm effect, Composite fermions PACS: 73.50.Jt, 73.40.Kp, 73.40.Lq, 73.40.Gk

The so-called angular magnetoresistance oscillations (AMRO) were originally discovered in the quasi-twodimensional (Q2D) organic conductors of the (BEDT$\mathrm{TTF})_{2} \mathrm{X}$ family $[1,2]$. Upon rotation of a magnetic field $\boldsymbol{B}$, electrical resistivity oscillates periodically in $\tan \theta$, where $\theta$ is the angle between $\boldsymbol{B}$ and the normal to the layers. The oscillations are very strong and the most pronounced in the interlayer resistivity $\rho_{z}$. AMRO are distinct from the Shubnikov-de Haas ( $\mathrm{SdH}$ ) oscillations, where resistivity oscillates as a function of the magnetic field magnitude for a fixed orientation. In AMRO, resistivity has maxima at certain angles $\theta$, often called the "magic angles", that are independent of the magnetic field magnitude. AMRO typically persist to substantially higher temperatures than the SdH oscillations, so the two effects can be clearly separated experimentally. Theory explained that the period of $\mathrm{AMRO}$ in $\tan \theta$ is inversely proportional to $k_{F} d$, where $d$ is the interlayer distance, and $k_{F}$ is the in-plane Fermi

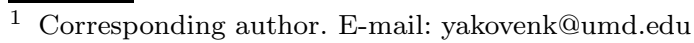

wave vector. Thus, AMRO can be utilized to determine $k_{F}$ and to map out Fermi surfaces of Q2D materials with anisotropic $k_{F}$. This was done first in $\beta$-(BEDT$\mathrm{TTF})_{2} \mathrm{IBr}_{2}$ [3], and then in a variety of organic conductors (see reviews $[4,5,6]$ ). AMRO were also observed in many other layered materials, such as intercalated graphite [7], $\mathrm{Sr}_{2} \mathrm{RuO}_{4}[8], \mathrm{Tl}_{2} \mathrm{Ba}_{2} \mathrm{CuO}_{6}[9,10]$, and the GaAs superlattices $[11,12,13]$.

The first theory of AMRO was presented by Yamaji [14], who pointed out that the amplitude of the SdH oscillations should be maximal at the magic angles determined by zeroes of the Bessel function $J_{0}\left(k_{F} d \tan \theta\right)$. Yagi et al. [15] calculated angular oscillations of the interlayer conductivity $\sigma_{z}(\theta)$ from the Boltzmann equation using semiclassical electron trajectories on the cylindrical 3D Fermi surface. It was assumed that a periodic crystal with many layers and a 3D Fermi surface is necessary for observation of AMRO. However, it was also recognized [15] that AMRO exist already in the limit of infinitesimal interlayer tunneling amplitude $t_{\perp} \rightarrow 0$. Using the Landau wave functions, Kurihara 

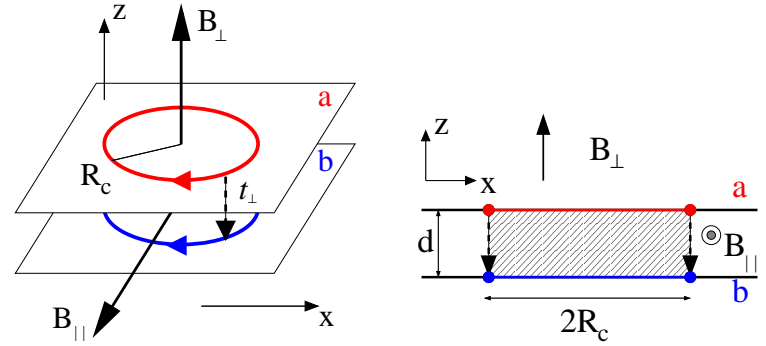

Fig. 1. (a) Tunneling geometry for a bilayer in a tilted magnetic field $\boldsymbol{B}$. The circles represent the quasiclassical cyclotron orbits. (b) View of the bilayer along the layers. Magnetic flux through the shaded area produces oscillations of the effective interlayer tunneling amplitude.

$[16,17]$ and Yoshioka [18] calculated the effective interlayer tunneling amplitude $\tilde{t}_{\perp}$ in a tilted magnetic field and found angular oscillations in $\tilde{t}_{\perp}(\theta)$. Then McKenzie and Moses $[19,20]$ explicitly demonstrated that electron tunneling between just two layers shows AMRO due to interference of the gauge phase differences between the layers. These ideas were further developed by Osada et al. for Q2D and Q1D materials [21,22].

Meanwhile, semiconducting bilayers were studied experimentally in parallel $[23,24]$ and tilted $[25,26]$ magnetic fields. On the theory side, $\mathrm{Hu}$ and MacDonald [27] calculated $\tilde{t}_{\perp}$ in a tilted field using the Landau wave functions, and Lyo et al. $[28,29,30]$ studied conductivity using the Kubo formula. They found vanishing $\tilde{t}_{\perp}$ for certain angles $\theta[27]$ and oscillatory dependence of $\sigma_{z}$ on the magnetic field component $B_{\|}$parallel to the layers for a fixed perpendicular component $B_{\perp}$ [29]. However, these papers (also [31]) focused on the low Landau filling factors, whereas Q2D metals were studied for the high filling factors, so a relation between AMRO in these two classes of materials was not recognized.

In this paper, we would like to make a connection between AMRO in layered metals and semiconducting bilayers. We present an intuitive geometrical interpretation of AMRO as the Aharonov-Bohm effect, both in real and momentum spaces. We start with the density-balanced bilayers, where both layers have the same Fermi surfaces, and then generalize to the density-imbalanced bilayers with different Fermi surfaces. We also speculate that it may be possible to observe AMRO when the layers are in the compositefermion state and to use AMRO for investigation of such a state. We hope that fresh insight from the or-

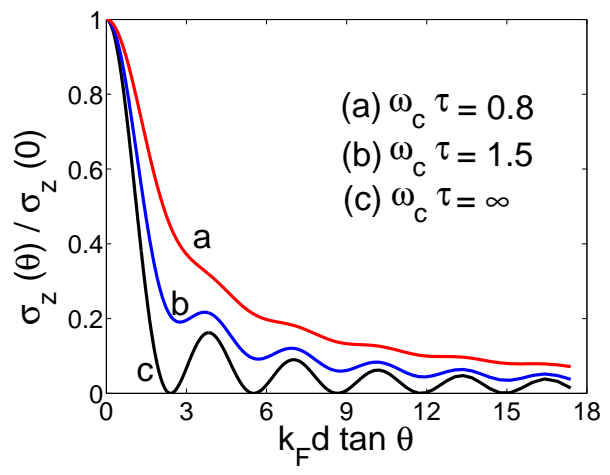

Fig. 2. Interlayer conductivity $\sigma_{z}$ calculated from Eq. (5) and plotted vs. $\tan \theta=B_{\|} / B_{\perp}$.

ganic conductors community will be stimulating for further studies of oscillatory phenomena in semiconductor bilayers (for the Q1D case see [32]).

The bilayer geometry is shown in Fig. 1. Electron tunneling between the layers $\mathrm{a}$ and $\mathrm{b}$ is described by the Hamiltonian

$\hat{H}_{\perp}=t_{\perp} \int \hat{\psi}_{a}^{\dagger}(\boldsymbol{r}) \hat{\psi}_{b}(\boldsymbol{r}) e^{\frac{i e A_{z}(\boldsymbol{r}) d}{\hbar c}} d^{2} r+$ H.c.,

where we have chosen the gauge $A_{z}=B_{\|} x$. We will assume that the interlayer tunneling amplitude $t_{\perp}$ is small compared with the intralayer energy scales, so it can be treated as a perturbation. We will use a quasiclassical approximation to describe the in-plane electron motion, assuming that the Landau filling factors are high enough. In the presence of $B_{\perp}$, electrons execute quasiclassical cyclotron motion within the layers with the frequency $\omega_{c}=e B_{\perp} / m$ and the radius $R_{c}=$ $c \hbar k_{F} / e B_{\perp}$. Here we used the Fermi wave vector $k_{F}$ in the formula for $R_{c}$, because only the electrons at the Fermi surface are relevant for conduction. For balanced bilayers, $k_{F}$ is the same in both layers.

The gauge phase in Eq. (1) leads to interference between electron tunneling at different points along the cyclotron orbit, and the effective tunneling amplitude $\tilde{t}_{\perp}$ is obtained by phase averaging $[19,20]$ :

$\tilde{t}_{\perp}=t_{\perp}\left\langle e^{\frac{i e B_{\|} x(t) d}{\hbar c}}\right\rangle_{t}=t_{\perp} J_{0}\left(k_{F} d \tan \theta\right)$.

Here the brackets represent averaging over time $t$ for the cyclotron motion $x(t)=R_{c} \cos \left(\omega_{c} t\right), J_{0}$ is the Bessel function, and $\tan \theta=B_{\|} / B_{\perp}$. Since the interlayer tunneling conductivity $\sigma_{z}$ is proportional to $\tilde{t}_{\perp}^{2}$, 
Eq. (2) gives $\sigma_{z}(\theta) / \sigma_{z}(0)=J_{0}^{2}\left(k_{F} d \tan \theta\right)$, which is shown by the curve (c) in Fig. 2. From the asymptotic expression $J_{0}(\xi) \propto \cos (\xi-\pi / 4) / \sqrt{\xi}$, we find that $\tilde{t}_{\perp}$ and $\sigma_{z}$ oscillate periodically in $\tan \theta$ and vanish at the "magic angles"

$\frac{B_{\|}}{B_{\perp}}=\tan \theta_{n}=\frac{\pi(n-1 / 4)}{k_{F} d}$,

where $n$ is an integer. This is the AMRO effect discussed in the introduction. In $[27,29,31]$, the effective tunneling amplitude $\tilde{t}_{\perp}$ was obtained as a matrix element of the Hamiltonian (1) between the Landau wave functions and expressed in terms of the Laguerre polynomials. However, as pointed out in Refs. [16,17,18], the Laguerre polynomials reduce to the Bessel function for the high Landau levels, so the quasiclassical expression (2) agrees with the quantum calculation [27,29,31].

Vanishing of $\tilde{t}_{\perp}$ at the magic angles not only results in minima of $\sigma_{z}$, but also in disappearance of beating in the $\mathrm{SdH}$ oscillations. Generally, the symmetric and antisymmetric electron states in a densitybalanced bilayer are split in energy by $\tilde{t}_{\perp}$, which results in two slightly different $\mathrm{SdH}$ frequencies. However, at the magic angles, the energy split and the beating of the $\mathrm{SdH}$ oscillations should disappear, because $\tilde{t}_{\perp} \rightarrow$ 0 . This effect is observed in organic conductors [5] and was explained theoretically by Yamaji [14]. In bilayers, it was observed [25] that the $\mathrm{SdH}$ beating period increases with the increase of $B_{\|}$, in qualitative agreement with the argument presented above. However, the ratio $B_{\|} / B_{\perp}$ was not big enough to reach a magic angle and to observe disappearance of the $\mathrm{SdH}$ beating.

AMRO can be interpreted geometrically as a particular manifestation of the Aharonov-Bohm effect. Let us look at the bilayer along the layers, as shown in Fig. 1b. The gauge phase in Eq. (1) is proportional to the area contained between the layers up to the point of electron tunneling. The lines of the length $2 R_{c}$ represent the side view of the cyclotron orbits. Electrons spend more time at the extremal turning points denoted as the dots, which naturally define the shaded area $2 R_{c} d$. The magnetic flux $\Phi$ through this area results in destructive interference between electron tunneling at the opposite turning points and vanishing of $\tilde{t}_{\perp}$ when $\Phi=2 R_{c} d B_{\|}=\phi_{0}(n+C)$, where $\phi_{0}=2 \pi \hbar c / e$ is the flux quantum, and $C$ is an appropriate constant. Inserting the expression for $R_{c}$, we recover Eq. (3). Notice that one dimension $d$ of the Aharonov-Bohm area
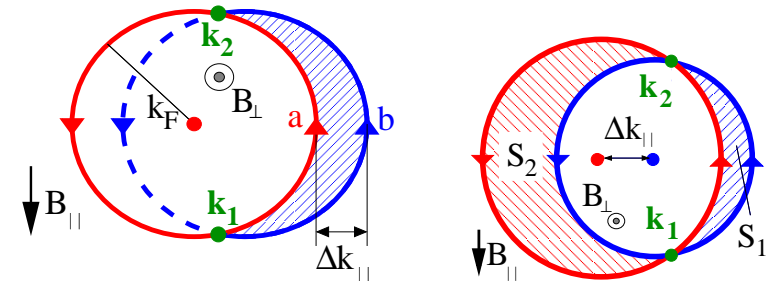

Fig. 3. Fermi surfaces of the layers in a bilayer that is (a) balanced and (b) imbalanced. The Fermi surfaces are displaced by $\Delta k_{\|}=e B_{\|} d / c$. The magnetic flux associated with the shaded areas in momentum space causes oscillations of the effective interlayer tunneling amplitude.

is fixed by the bilayer structure, but the other dimension $2 R_{c}$ is adjustable and is proportional to $B_{\perp}^{-1}$. This results in the condition (3) on the ratio of $B_{\|}$and $B_{\perp}$.

AMRO can be also interpreted as a result of interference in the momentum space, as illustrated in Fig. 3. Suppose that only the $B_{\|}$component is applied. Then, according to Eq. (1), the in-plane electron momentum changes by $\Delta k_{\|}=e B_{\|} d / c \hbar$ upon tunneling between the layers $[23,24,25]$, so the Fermi surfaces of the two layers are shifted relative to each other as shown in Fig. 3. Thus, electrons can tunnel only at the points $\boldsymbol{k}_{1}$ and $\boldsymbol{k}_{2}$, where the conservation laws of both energy and momentum are satisfied. When the $B_{\perp}$ component is turned on, it causes interference between the two trajectories a and b connecting the points $\boldsymbol{k}_{1}$ and $\boldsymbol{k}_{2}$. The phase difference between the two trajectories is proportional to the shaded area $S$ between them in momentum space. In the balanced case shown in Fig. $3 \mathrm{a}, S \approx$ $2 k_{F} \Delta k_{\|}$, where we assumed that $\Delta k_{\|} \ll k_{F}$, which is a typical condition for Q2D metals. The interference between the two momentum-space trajectories is destructive when the condition $B_{\perp}=\phi_{0} S /(n+C)(2 \pi)^{2}$ is satisfied, which reproduces Eq. (3).

In the imbalanced case, the interference oscillations develop between the parallel trajectories that involve the momentum-space areas $S_{1}$ and $S_{2}$ in Fig. 3b. The frequencies of these oscillations are given by the $\mathrm{SdH}$ like formula $B_{\perp}=\phi_{0} S_{1,2} /(n+C)(2 \pi)^{2}$, where the areas $S_{1}$ and $S_{2}$ depend on $B_{\|}$. Notice that these interference oscillations are different from the SdH oscillations. The later are the consequence of the energy quantization originating from closed orbits, whereas the former result from quantum interference between parallel orbits that do not form a closed loop and do not produce energy quantization. Magnetoresistance oscil- 
lations due to the momentum-space interference are known in some metals [33] and organic conductors [34]. The in-plane resistivity $\sigma_{x}$ of an imbalanced bilayer in tilted magnetic fields was measured in Ref. [26]. The oscillations originating from the areas $S_{1}$ and $S_{2}$ can probably be found in the Fourier spectrum shown in Fig. 4 of Ref. [26]. However, this paper focused only on the $\mathrm{SdH}$ oscillations originating from closed orbits, but not on the interference oscillations from parallel orbits. In Fig. 3 of this paper, one can recognize a pattern of lines at certain angles $\tan \theta=B_{\|} / B_{\perp}$, which can be interpreted as observation of AMRO. It would be very interesting to measure the interlayer conductivity $\sigma_{z}$, where the AMRO effect should be stronger than in $\sigma_{x}$.

A finite lifetime $\tau$ of quasiparticles results in loss of phase coherence, which can be described phenomenologically by an exponentially decaying factor in the Kubo formula for $\sigma_{z}[15,19,20]$ :

$\sigma_{z} \propto t_{\perp}^{2}\left\langle\int_{t}^{\infty} e^{\frac{i e B_{\|} d}{\hbar c}\left[x(t)-x\left(t^{\prime}\right)\right]} e^{-\frac{t^{\prime}-t}{\tau}} d t^{\prime}\right\rangle_{t}$.

Doing the integral in Eq. (4), one finds [15,19,20]

$\frac{\sigma_{z}(\boldsymbol{B})}{\sigma_{z}(0)}=J_{0}^{2}\left(k_{F} d \tan \theta\right)+2 \sum_{j=1}^{\infty} \frac{J_{j}^{2}\left(k_{F} d \tan \theta\right)}{1+\left(j \omega_{c} \tau\right)^{2}}$

For $\omega_{c} \tau \gg 1$, Eq. (4) gives $\sigma_{z} \propto \tilde{t}_{\perp}^{2} \tau$, and Eq. (5) reproduces AMRO. However, for $\omega_{c} \tau \ll 1$, electrons lose coherence before they complete a cycle, so the interference effect is washed out, and $\sigma_{z}$ reduces to $\sigma_{z}(0) \propto$ $t_{\perp}^{2} \tau$. Fig. 2 shows $\sigma_{z}(\theta)$ calculated from Eq. (5) for several values of $\omega_{c} \tau$. When $B$ is increased at a fixed angle $\theta$, resistivity $\rho_{z}=1 / \sigma_{z}$ increases and saturates at a finite value in the limit $\omega_{c} \tau \rightarrow \infty$ for generic angles. However, for the magic angles, $\rho_{z}$ increases without saturation, because $\sigma_{z} \rightarrow 0$ at $\omega_{c} \tau \rightarrow \infty$. Notice that observation of AMRO requires $\omega_{c} \tau>1$, whereas, according to the Lifshitz-Kosevich formula, observation of the $\mathrm{SdH}$ oscillations requires $\hbar \omega_{c}>T$, where $T$ is temperature. These are different conditions, and, typically, AMRO are still visible at elevated temperatures, where the $\mathrm{SdH}$ oscillations have already disappeared. For example, in GaAs superlattices [12], AMRO are clearly visible at $25 \mathrm{~K}$, whereas the $\mathrm{SdH}$ oscillations dominate at $1.5 \mathrm{~K}$.

Finally, we briefly discuss a possibility of observing AMRO in the case where each layer of a bilayer is in the composite-fermion state with the filling factor $\nu$ close to $1 / 2$. The composite fermions experience the effective magnetic field $B_{\perp}^{*}=B_{\perp}(1-2 \nu)$ and execute cyclotron motion with the radius $R_{c}^{*}=k_{F}^{*} \phi_{0} / 2 \pi B_{\perp}^{*}$, where $k_{F}^{*}=$ $\sqrt{2} k_{F}$ is their effective Fermi wave vector. By analogy, we would expect to see AMRO in the interlayer conductivity with the magic angles given by Eq. (3) with the substitution $B_{\perp} \rightarrow B_{\perp}^{*}$ and $k_{F} \rightarrow k_{F}^{*}$. Unfortunately, the interlayer tunneling is greatly suppressed, because the composite fermions need to decompose and recompose for tunneling [35]. However, the interlayer conductivity may increase at higher temperatures and help to observe AMRO. A systematic attempt to observe AMRO would provide useful information about the nature of the composite-fermion state.

\section{References}

[1] M.V. Kartsovnik et al. JETP Lett. 48 (1988) 541.

[2] K. Kajita et al. Sol. St. Comm. 70 (1989) 1189.

[3] M.V. Kartsovnik et al. J. Phys. I (France) 2 (1991) 89.

[4] M. V. Kartsovnik, V. N. Laukhin, J. Phys. I France 6 (1996) 1753.

[5] J. Wosnitza et al. J. Phys. I France 6 (1996) 1597.

[6] J. Singleton, Rep. Prog. Phys. 63 (2000) 1111.

[7] Y. Iye et al. J. Phys. Soc. Jpn., 63 (1994) 1643.

[8] E. Ohmichi et al. Phys. Rev. B 59 (1999) 7263.

[9] A. Dragulescu et al. Phys. Rev. B 60 (1999) 6312.

[10] N.E. Hussey et al. Nature 425 (2003) 814.

[11] R. Yagi et al. J. Phys. Soc. Jpn. 60 (1991) 3784.

[12] M. Kawamura et al. Physica B 249-251 (1998) 882.

[13] T. Osada et al. Physica B 294-295 (2001) 402.

[14] K. Yamaji, J. Phys. Soc. Jpn. 58 (1989) 1520.

[15] R. Yagi et al. J. Phys. Soc. Jpn. 59 (1990) 3069.

[16] Y. Kurihara, J. Phys. Soc. Jpn. 61 (1992) 975.

[17] Y. Kurihara, J. Phys. Soc. Jpn. 62 (1993) 255.

[18] D. Yoshioka, J. Phys. Soc. Jpn. 64 (1992) 3168.

[19] R.H. McKenzie, P. Moses, Phys. Rev. Lett. 81 (1998) 4492.

[20] P. Moses, R.H. McKenzie, Phys. Rev. B 60 (1999) 7998.

[21] T. Osada, Physica E 12 (2002) 272.

[22] T. Osada et al. Physica E 18 (2003) 200.

[23] J.P. Eisenstein et al. Phys. Rev. B 44 (1991) 6511.

[24] J.A. Simmons et al. Phys. Rev. B 47 (1993) 15741.

[25] G.S. Boebinger et al. Phys. Rev. B 43 (1991) 12673.

[26] N.E. Harff et al. Phys. Rev. B, 55 (1997) R13405.

[27] J. Hu, A.H. MacDonald, Phys. Rev. B 46 (1992) 12554.

[28] S.K. Lyo, J.A. Simmons, J. Phys.: Condens. Matter 5 (1993) L299.

[29] S.K. Lyo, Phys. Rev. B 57 (1998) 9114.

[30] S.K. Lyo et al. Phys. Rev. B 58 (1998) 1572.

[31] R. Côté et al. Phys. Rev. B 66 (2002) 205315. 
[32] B.K. Cooper, V.M. Yakovenko, Phys. Rev. Lett. 96 (2006) 037001.

[33] C.B. Friedberg, R.W. Stark, Low Temp. Phys. (LT-13) 4 (1974) 177.

[34] X. Yan et al. Synth. Metals 27 (1988) 145.

[35] I.B. Spielman et al. Phys. Rev. B 70 (2004) 081303. 